\title{
In-hospital mortality rate after osteoporotic hip fracture in Bucharest
}

\author{
Ramona Dobre*, Dan Niculescu***, Gheorghe Popescu******, Adrian Barbilian*******, \\ Cătălin Cîrstoiu*****, Cătălina Poiană*** \\ *Department of Hypophysis and Neurohypophysis, "C. I. Parhon" National Institute of Endocrinology, \\ Bucharest, Romania \\ **"Carol Davila" University of Medicine and Pharmacy, Bucharest, Romania \\ $* * *$ Emergency University Hospital, Bucharest, Romania \\ $* * * *$ Clinical Emergency Hospital, Bucharest, Romania \\ $* * * * *$ "Dr. Carol Davila" Central Military Emergency University Hospital, Bucharest, Romania
}

Correspondence to: Dobre Ramona, MD, "Carol Davila" University of Medicine and Pharmacy, Bucharest, Romania 9 Emil Gârleanu Street, Bucharest Mobile phone: +400770597590, E-mail: savinramonago@gmail.com

\begin{abstract}
Introduction: Hip fracture is the most severe consequence of osteoporosis and an important cause of excess mortality in the elderly.

Objective: We aimed to evaluate the in-hospital mortality rate after osteoporotic hip fracture in patients treated surgically or functionally in specialized centers in Bucharest.

Materials and methods: We calculated the in-hospital mortality rate in 745 patients (540 women [72.48\%], with a mean age of $79.1 \pm 11$ years), surgically or functionally treated for fragility hip fracture over a 12 months period.

Results: Average length of hospitalization was 18.12 days. In hospital mortality rate was 5.36\% ( $n=40$, women 60\%). An important risk factor associated with mortality was age, $p=0.001$. The male sex was also a risk factor with a mortality rate of $7,8 \%(n=16)$, compared to $4.44 \%$ in women, $p<0.005$, with OR of 1.57 . Out of the 40 patients, $57.5 \%$ had a femoral neck fracture, 35\% intertrochanteric, and 5.5\% atypical fracture in absence of bisphosphonates. $7.5 \%$ had previous fragility fractures. $85 \%$ of the patients had a history of one or more cardiac pathologies (34.28\% with atrial fibrillation), $57.5 \%$ underwent surgical intervention $(n=23)$ with an average day of intervention of 8.82 after admission. None of the patients had an osteoporosis treatment before the event and on average 3.73 medications with an increased risk of falling and fracture.

Conclusion: In-hospital mortality rate after hip fracture remains high; probably this being related to the high comorbidity associated with male sex and increased age as risk factors.

Keywords: osteoporosis, hip fracture, mortality, in-hospital
\end{abstract}

\section{Introduction}

Osteoporosis is a disorder characterized by low bone mass and microarchitectural disruption that results in bone fragility and risk of fractures with minimal trauma (less or equal that a fall from a standing position). Elderly population is the fastest growing population worldwide and the incidence of fragility fracture is expected to rise substantially but even if it remained stable it is estimated to rise from 1.7 million in 1990 to 6.3 in 2050 [1]. Annually, in Romania, it is estimated that almost 100.000 fragility fractures occur, 15.000 being located 
on the hip and being sustained, thus making our country a moderate risk country for the hip fracture [2].

Hip fracture remains the most important clinical manifestation of osteoporosis, with a high and relatively unchanged mortality rate in the last decades [3]. In-hospital mortality is an important statistical measure, being used in some countries as a reliable quality indicator of healthcare.

To our knowledge, there are not any studies regarding the mortality rate after hip fracture in Romania [4].

In this study, we aimed to evaluate the in-hospital mortality rate after osteoporotic hip fracture in patients treated surgically or functionally in specialized centers in Bucharest, to compare it to the data existing in literature and to look for associated predictive factors of increased mortality risk.

\section{Patients and methods}

We calculated the in-hospital mortality rate after hip fracture in 745 patients (540 women [72.48\%], with a mean age of $79.1 \pm 11$ years) admitted in 3 trauma centers in Bucharest, from 01.09.2017 to 31.08.2018.

Patients with the home addresses in Bucharest were $52.5 \%$ and $66.71 \%$ from urban areas. We selected retrospectively all the patients with $\mathrm{S}_{72.0 \mathrm{x}} \mathrm{S}_{72.1 \mathrm{x}}$ and $\mathrm{S}_{72.2 \mathrm{x}}$, S72.3X (femoral neck, intertrochanteric, subtrochanteric, subtrochanteric-diaphyseal coded as only diaphyseal) from the electronic databases of the selected centers. All charts were reviewed for data validation. The rest of the data (address, medical history, treatment, day of admission after fracture, type of surgery, day of the intervention, and diagnoses) was also collected from the patient's charts (physical and electronic in $86.5 \%$ and only electronic chart in $13.5 \%$ ).

We excluded patients younger than 40 years old, high intensity trauma fractures, and pathological fractures, even when suspicion existed with no further investigations.

For data analysis, Microsoft Excel 16.16.8, 2016 was used.

\section{Results}

In-hospital mortality rate was 5.36\% ( $\mathrm{n}=40$, women 60\%).

An important risk factor associated with mortality was age, $p=0.001$.

The male sex was also a risk factor with a mortality rate of $7,8 \%(n=16)$, compared to $4.44 \%$ in women, $\mathrm{p}<0.005$, with OR of 1.57.

Out of the 40 patients, $57.5 \%$ had a femoral neck fracture, $35 \%$ intertrochanteric, and $5.5 \%$ atypical fracture in the absence of bisphosphonates, percentages that were slightly different in the whole population studied, with a mortality rate of $6.4 \%$ for the femoral neck fracture compared to $4.9 \%$ for the other types, with OR of 1.3.

$57.5 \%$ sustained surgical interventions $(n=23)$ with a mortality rate of $3.6 \%$ in surgically treated patients compared to $16 \%$ in the functionally treated group. The average day of intervention of 8.82 after admission compared to 3.61 in the patients who survived, made the postponing of the surgical intervention a risk factor, $p=0.007$. The average length of hospitalization was 18.12 days compared to 11.68 days per patient in the survival group.

$85 \%$ of the patients had a history of one or more cardiac pathologies $(34.28 \%$ with atrial fibrillation) compared to $82.12 \%$ in the other group. Also, when talking about respiratory disorders, the prevalence was $7.5 \%$ compared to $9.2 \%$ in the survival group. History of prevalent fracture was present in $17.26 \%$ of the patients included with $56.25 \%$ located also at the hip level. $10 \%$ of the patients who died had a prevalent fracture, with $50 \%$ located at the hip level.

\section{Discussions}

The in-hospital mortality rate in our study was $5.36 \%$, consistent with the reported rates 
published from 2013 to 2018: Belmont et al. [5] reported a $4.5 \%$ mortality rate in 9286 cases, Frost et al. [6] a 6\% rate in 1504 cases, Alzahrani et al. [7] a 5\% rate in 2178 cases, Chatterton et al. [8] a $6.5 \%$ rate in 4426 cases, Johansen et al. [9] a 5.2\% rate in 1050 fractures, SanchezHernandez et al. [10] a 5.1\% rate in 216 hip fractures treated functionally, Mesa-Lampré et al. [11] published a $6.9 \%$ rate in 494 hip fractures managed in an orthogeriatrics unit.

As an unmodifiable risk factor for mortality after hip fracture, male sex has been demonstrated on several publications, like Panula J. et al. [12] with HR of 1.55 and Kannegaard PN et al. [13] with HR of 1.7. Our study showed this relationship too, with OR of 1.57, $p<0.005$. Male sex and increasing age are included in most of the predicting models like Nottingham Hip Fracture score or National Hip Fracture Database case-mix adjustment model in UK [14].

Although most of the studies regarding osteoporotic hip fracture include only femoral neck and intertrochanteric fracture, the number of subtrochanteric ones was very small and we preferred to include them.

Regarding the average day of the intervention, we could observe that patients who died during hospital admission were operated later than the survival group. $20 \%$ of the patients who died during hospitalization had anemia compared to $7.9 \%$ in the survival group and the proportion of patients on anticoagulation therapy in the patients that did not survive was $27.5 \%$ compared to $1.27 \%$ patients discharged. The optimum timing of surgery for a hip fracture is an important topic for researchers but still with no clear consensus. It is generally accepted that early surgery is beneficial $[15,16]$, the more fitted patients can benefit from it but the frail patients need a delay for correction of eventual surgical risk factors like anemia, anticoagulation or electrolyte imbalance [17].

It is very hard to state if functionally treated fractures are at an increased risk of mortality, these patients already presenting a high presurgical mortality risk that contraindicated the surgery. Only one patient refused the surgery and 3 of them had an extremely severe state.

It is necessary to expand the study to observe the impact of functionally treated fractures versus surgically treated ones on the 30 days and 12 months mortality rate.

We need further studies to expand the evaluation of the mortality rate to 30 days and even 12 months after the hip fracture. It is important to assess how much of the inhospital mortality rate contributes to the 30 days mortality rate.

The data raised questions about the relationship between the surgical timing delay and the mortality rate. We need further studies to see what the causes of surgical timing delay in our hospitals are, to differentiate from other problems like availability of resources, and intervene if possible.

Further studies are necessary to separately assess the subtrochanteric, diaphyseal and distal femur, and to search for common characteristics and to compare the mortality rate and the risk factors of mortality with the age-related osteoporosis hip fractures [18].

The limitations of the study reside in the lack of uniformity in our data collecting systems and the ICD-10 codes. Unfortunately, regarding comorbidities data and treatment history, this type of study has many limitations and depends on the medical staff that is responsible for the patient chart. A prospective study is indicated.

\section{Conclusions}

Mortality rate after hip fracture remains high but relatively similar to the one in literature data. We managed to validate some of the mortality risk factors present in published literature. 


\section{Conflict of Interest statements}

Authors state no conflict of interest.

\section{Informed Consent and Human and Animal}

\section{Rights statements}

Informed consent has been obtained from all individuals included in this study.

\section{Authorization for the use of human subjects}

Ethical approval: The research related to human use complies with all the relevant national regulations, institutional policies, is in accordance with the tenets of the Helsinki Declaration, and has been approved by the authors' institutional review board or equivalent commitee.

\section{References}

1. Grigorie D, Sucaliuc A, Johansson H, Kanis JA, McCloskey E. Incidence of hip fracture in Romania and the development of a Romanian FRAX model. Calcif Tissue Int. 2013 May; 92(5):429-36.

2. Poiana C, Fica S. Endocrinologie pentru studenti si rezidenti, 2015, Bucuresti, 181-195.

3. Abrahamsen B, van Staa T, Ariely R, Olson M, Cooper C. Excess mortality following hip fracture: a systematic epidemiological review. Osteoporos Int. 2009 Oct; 20(10):1633-50.

4. Giannoulis D, Calori GM, Giannoudis PV. Thirty-day mortality after hip fractures: has anything changed?. Eur J Orthop Surg Traumatol. 2016; 26(4):365-370.

5. Belmont P, Garcia E, Romano D. Risk factors for complications and in-hospital mortality following hip fractures: a study using the National Trauma Data Bank. Arch Orthop Trauma Surg. 2014; 134:597-604.

6. Frost SA, Nguyen ND, Black DA. Risk factors for inhospital post-hip fracture mortality. Bone. 2011; 49:553-558.

7. Alzahrani K, Gandhi R, Davis A. In-hospital mortality following hip fracture care in southern Ontario. Can J Surg. 2010; 53:294-298.

8. Chatterton BD, Moores TS, Ahmad S. Cause of death and factors associated with early in-hospital mortality after hip fracture. Bone Joint J. 2015; 97-B:246-251.

9. Johansen A, Mansor M, Beck S. Outcome following hip fracture: post-discharge residence and long-term mortality. Age Ageing. 2010; 39:653-656.

10. Sánchez-Hernández N, Sáez-López P, Paniagua-Tejo S. Results following the implementation of a clinical pathway in the process of care to elderly patients with osteoporotic hip fracture in a second level hospital. Rev Esp Cir Ortop Traumatol. 2016; 60:1-11.

11. Mesa-Lampré MP, Canales-Cortés V, Castro-Vilela ME. Initial experiences of an orthogeriatric unit. Rev Esp Cir Ortop Traumatol. 2015; 59:429-438.

12. Panula J, Pihlajamäki H, Mattila VM et al. Mortality and cause of death in hip fracture patients aged 65 or older: a population-based study. BMC Musculoskelet Disord. 2011; $12: 105$.

13. Kannegaard PN, van der Mark S, Eiken P, Abrahamsen B. Excess mortality in men compared with women following a hip fracture. National analysis of comedications, comorbidity and survival. Age Ageing. 2010 Mar; 39(2):203-9.

14. Tsang C, Boulton C, Burgon V, Johansen A, Wakeman R, Cromwell DA. Predicting 30-day mortality after hip fracture surgery: Evaluation of the National Hip Fracture Database case-mix adjustment model. Bone Joint Res. 2017 Sep;6(9):550-556

15. Khan SK, Kalra S, Khanna A, Thiruvengada MM, Parker MJ. Timing of surgery for hip fractures: a systematic review of 52 published studies involving 291, 413 patients. Injury. 2009; 40(7):692-697.

16. Carretta E, Bochicchio V, Rucci P, Fabbri G, Laus M, Fantini MP. Hip fracture: effectiveness of early surgery to prevent 30-day mortality. Int Orthop. 2010; 35(3):419424.

17. nice.org.uk/NICE guidance for hip fracture management.

18. Ng AC, Drake MT, Clarke BL et al. Trends in subtrochanteric, diaphyseal, and distal femur fractures, 1984-2007. Osteoporos Int. 2011; 23(6):1721-1726. 